\title{
CORRECTION
}

Check for updates

Cite this: Soft Matter, 2021, 17, 3250

DOI: $10.1039 / \mathrm{d} 1 \mathrm{sm} 90036 \mathrm{f}$

rsc.li/soft-matter-journal

\section{Correction: Collective motion of chiral Brownian particles controlled by a circularly-polarized laser beam}

\author{
Raúl Josué Hernández, (D) *a Francisco J. Sevilla, (D) ${ }^{\mathrm{b}}$ Alfredo Mazzulla, (D) ${ }^{\mathrm{c}}$ \\ Pasquale Pagliusi, (DD ${ }^{c d}$ Nicola Pellizzid ${ }^{d}$ and Gabriella Cipparrone $\mathbb{D}^{\text {(D) }}$
}

Correction for 'Collective motion of chiral Brownian particles controlled by a circularly-polarized laser beam' by Raúl Josué Hernández et al., Soft Matter, 2020, 16, 7704-7714, DOI: 10.1039/C9SM02404B.

The authors regret that the rotation sense of the $\mathscr{L}$-type and $\mathscr{R}$-type particles has been reversed in the video, figures and text, due to an artefact introduced by the video processing. Therefore, they would like to correct the following errors:

- At the beginning of Section 3, the sentence "When three $\mathscr{L}$-type particles are illuminated with LCP light, they exhibit an intermittent counterclockwise (ccw) circular motion..." should instead read as follows: "When three $\mathscr{L}$-type particles are illuminated with LCP light, they exhibit an intermittent clockwise (cw) circular motion..."

- On page 7706, right column, line 10, the sentence "while RCP light now induces a collective vortex rotation in the cw sense..." should instead read as follows: "while RCP light now induces a collective vortex rotation in the ccw sense..."

- In the Fig. 3 caption, the sentence "...counterclockwise rotation (ccw)..." should instead read as follows: “. . clockwise rotation $(\mathbf{c w}) \ldots$,

- In the Fig. 4 caption, the sentence "... clockwise rotation $(\mathbf{c w}) \ldots$.." should instead read as follows: "...counterclockwise rotation $(\mathbf{c c w}) . . . "$

- In the paragraph 3.1 "Angular Momentum", the sentence “... in agreement with the clockwise (cw) cooperative vortical motion..." should instead read as follows: "... in agreement with the counterclockwise (ccw) cooperative vortical motion..."

- Videos 1-4 in the ESI have been corrected.

Corrections to Fig. 3, 4 and 6 are minimal as shown below:

\footnotetext{
${ }^{a}$ Cátedra CONACYT Consejo Nacional de Ciencia y Tecnología - Instituto de Ciencias Nucleares, Universidad Nacional Autónoma de México, Apdo. Postal 70-543, 04510 Cd. de México, Mexico.E-mail: josue.hernandez@correo.nucleares.unam.mx

${ }^{b}$ Instituto de Física, Universidad Nacional Autónoma de México, Apdo. Postal 20-364, 01000 Cd. de México, Mexico

${ }^{c}$ CNR-Nanotec, UOS Cosenza, Ponte P. Bucci, Cubo 33B, 87036 Rende (CS), Italy

${ }^{d}$ Dipartimento di Fisica, Università della Calabria, Ponte P. Bucci, Cubo 33B, 87036 Rende (CS), Italy. E-mail: gabriella.cipparrone@fis.unical.it
} 

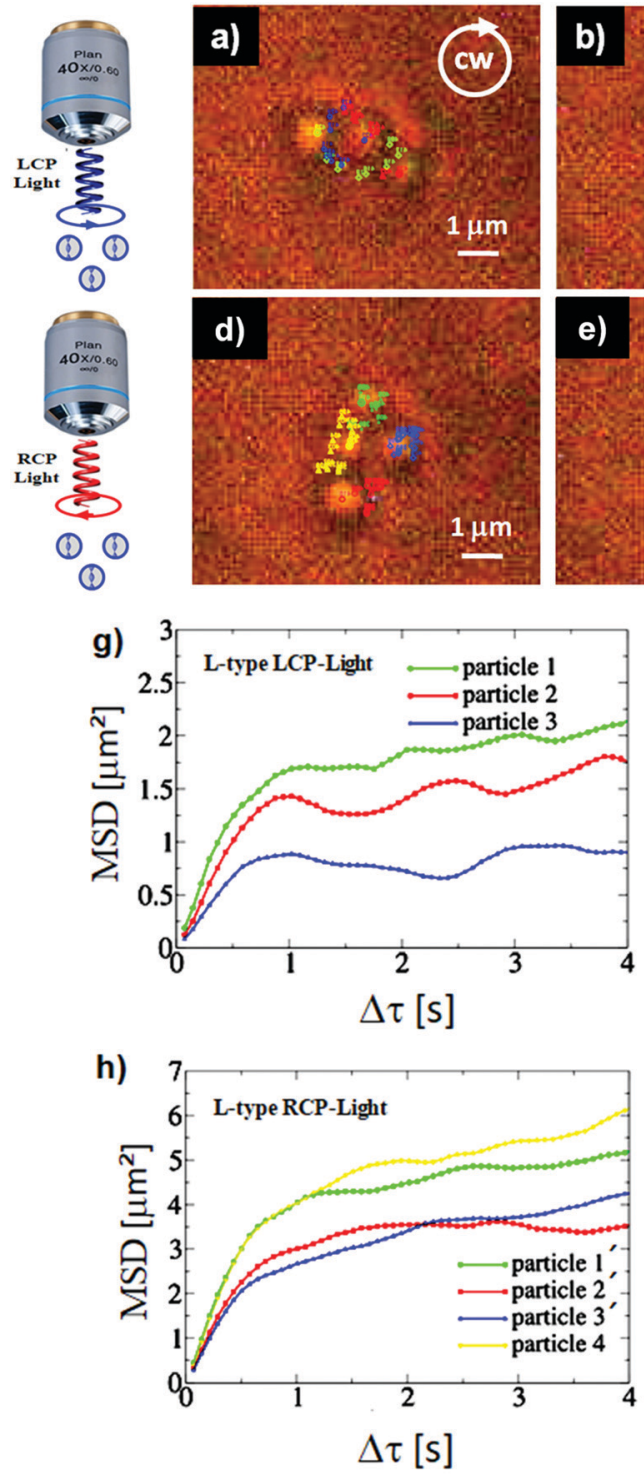
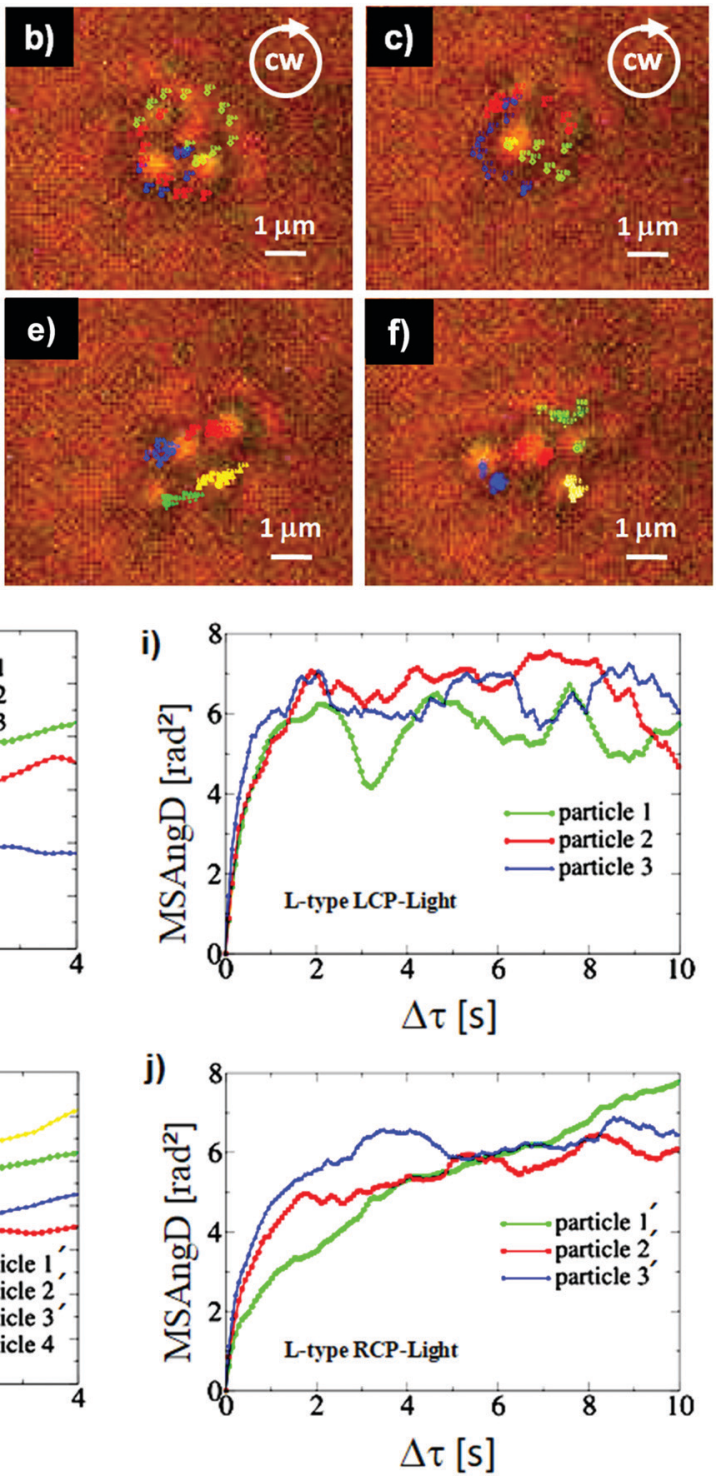

Fig. 3 Three types of chiral particles; each frame shows the last 15 steps in time $(\Delta t=0.071 \mathrm{~s})$, indicating the position of each particle by color: green (particle 1), red (particle 2) and blue (particle 3). (a)-(c) Image sequence for LCP light at $t_{\mathrm{a}}=10.2 \mathrm{~s} ; t_{\mathrm{b}}=21.8 \mathrm{~s}$, and $t_{\mathrm{c}}=31.1 \mathrm{~s}$, clockwise rotation (cw) and mean orbital radius $r=1.15 \pm 0.18 \mu \mathrm{m}$ (see Video 1, ESI $\dagger$ ). (d)-(f) Mean square displacement graphic for each particle; image sequence for the same particles now illuminated by RCP light at $t_{d}=10.2 \mathrm{~s}, t_{\mathrm{e}}=21.3 \mathrm{~s}$, and $t_{\mathrm{f}}=31.0 \mathrm{~s}$ (see Video 2, ESI + ). Mean square displacement graphics for each particle with (g) LCP and (h) RCP light and their corresponding mean square angular displacement graphics (i) and (j). 

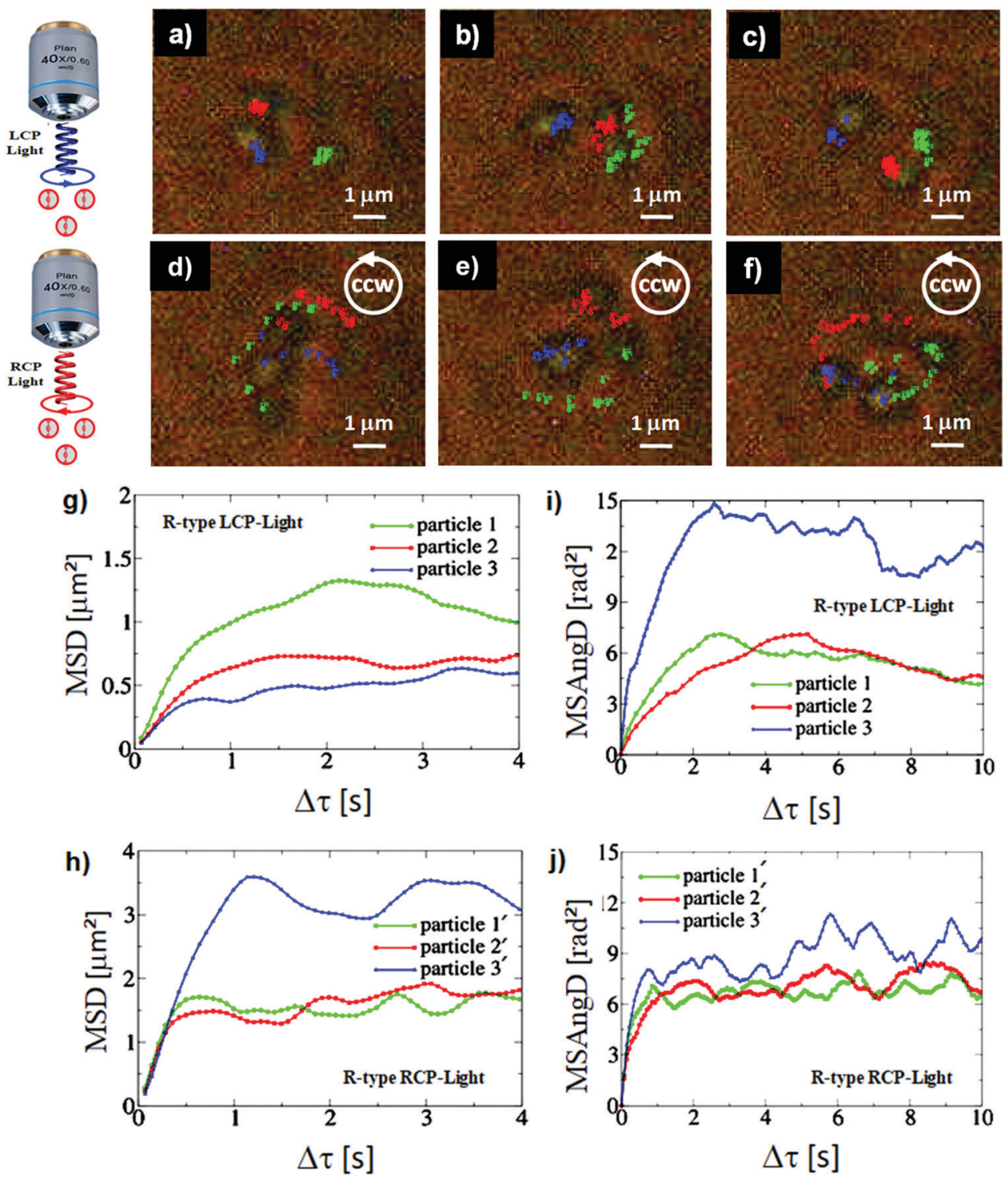

Fig. 4 Three $R$-type chiral particles; each frame shows the last 15 steps in time $(\Delta t=0.071 \mathrm{~s})$, indicating the position of each particle by color: green (particle 1), red (particle 2) and blue (particle 3). (a)-(c) Image sequence for LCP light at $t_{\mathrm{a}}=10.1 \mathrm{~s} ; t_{\mathrm{b}}=19.7 \mathrm{~s}$, and $t_{\mathrm{c}}=26.6 \mathrm{~s}$ (see Video 3 , ESI $\dagger$ ). (d) -(f) Mean square displacement graphic for each particle; image sequence for the same particles now illuminated by RCP light at $t_{\mathrm{d}}=10.8 \mathrm{~s}, t_{\mathrm{e}}=19.6 \mathrm{~s}$, and $t_{\mathrm{f}}=26.8 \mathrm{~s}$, counterclockwise rotation (ccw) and mean orbital radius $r=1.47 \pm 0.28 \mu \mathrm{m}$ (see Video 4, ESI $\dagger$ ). Mean square displacement graphics for each particle with (g) LCP and (h) RCP light and their corresponding mean square angular displacement graphics (i) and (j). 


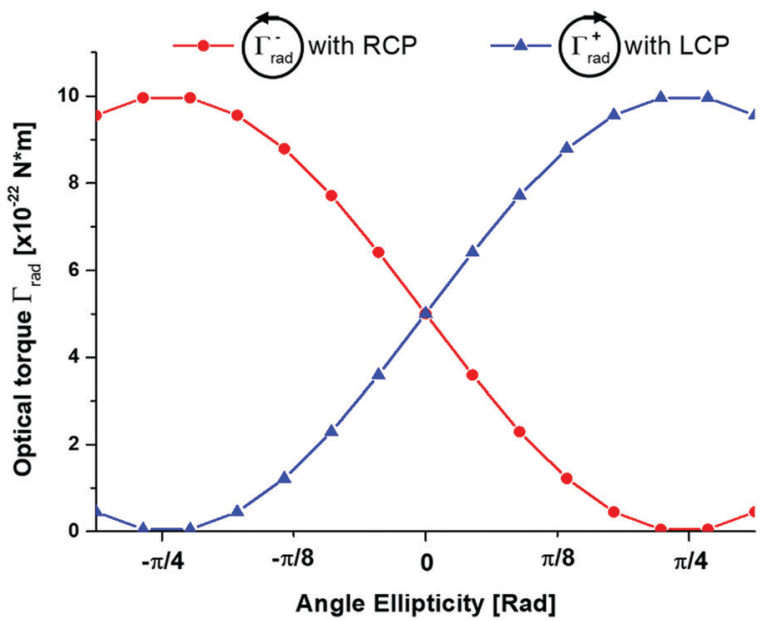

Fig. 6 Optical torque on a single particle $\vec{\Gamma}_{\text {rad }}$ with opposite reflectivities $R^{+}, R^{-}$versus ellipticity angle $\varphi$. With radius $a=500 \mathrm{~nm}$ and pitch $p=330 \mathrm{~nm}$ at $\lambda=488 \mathrm{~nm}, P=20 \mu \mathrm{W}$ and $R^{ \pm}=0.10$

The Royal Society of Chemistry apologises for these errors and any consequent inconvenience to authors and readers. 